\title{
SEROPREVALENCE OF LYME DISEASE IN EASTERN SLOVAKIA
}

\author{
Andrea Bušová1, Erik Dorko1, Kvetoslava Rimárová1, Jana Diabelková1, Tímea Rovenská1, Eva Feketeová2, \\ Matúš Bereš ${ }^{3}$, Róbert Čellár ${ }^{3}$, Zuzana Baranová ${ }^{4}$, Tomáš Kampe ${ }^{4}$, Karim Benhatchi ${ }^{5}$ \\ ${ }^{1}$ Department of Public Health and Hygiene, Faculty of Medicine, Pavol Jozef Šafárik University in Košice, Košice, Slovak Republic \\ 2Department of Neurology, Faculty of Medicine, Pavol Jozef Šafárik University in Košice and Louis Pasteur University Hospital, Košice, \\ Slovak Republic \\ ${ }^{3}$ Department of Orthopaedics and Traumatology of Locomotory Apparatus, Faculty of Medicine, Pavol Jozef Šafárik University in Košice \\ and Louis Pasteur University Hospital, Košice, Slovak Republic \\ ${ }^{4}$ Department of Dermatovenerology, Faculty of Medicine, Pavol Jozef Šafárik University in Košice and Louis Pasteur University Hospital, Košice, \\ Slovak Republic \\ ${ }^{5} 1$ st Department of Internal Medicine, Faculty of Medicine, Pavol Jozef Šafárik University in Košice and Louis Pasteur University Hospital, \\ Košice, Slovak Republic
}

\section{SUMMARY}

Objective: Lyme disease (LD) is chronic, multi-system zoonosis transmitted by ticks, and LD aetiological agents are spirochetes of the Borrelia burgdorferi sensu lato complex. The aim of the cross-sectional study was to analyze the LD incidence on the basis of the presence of specific antibodies in the serum of patients in Eastern Slovakia, and to compare the results of serological ELISA and immunoblot assays.

Methods: Venous blood with questionnaires was obtained by field sampling of respondents from Eastern Slovakia. Overall, we examined 537 human sera by the ELISA and for confirmation we tested all positive lgG antibodies against the Borrelia immunoblot assay.

Results: Our results confirmed the high serum prevalence of anti-Borrelia antibodies (17.9\% for $\lg \mathrm{G})$, while the immunoblot seropositive test was confirmed in $69.8 \%$ of responders from ELISA IgG positive sera. Positive antibodies of the IgM class were found in $7.6 \%$ of the population under study. Most commonly found were antibodies against VISE (80.2\%), p41 (66.7\%), p18 (56.3\%), p100 (41.7\%), p58 (31.3\%), and p39 (30.2\%).

Conclusion: It should be noted that detection of antibodies against $B$. burgdorferi .l. is only an indirect evidence of the presence of this bacterium in the development of clinical signs of $L D$ in humans. Laboratory $L D$ tests should be performed in accordance with valid standards, positive and uncertain results must be confirmed by the Western Blot/lmmunoblot assay.

Key words: Lyme disease, diagnosis, seroprevalence

Address for correspondence: E. Dorko, Department of Public Health and Hygiene, Faculty of Medicine, Pavol Jozef Šafárik University in Košice, Šrobárova 2, 04180 Košice, Slovak Republic. E-mail: erik.dorko@upjs.sk

https://doi.org/10.21101/cejph.a5442

\section{INTRODUCTION}

Lyme disease (LD) has a global reach and has become a major problem in diagnostics and therapy in current medicine and is a significant public health problem in the endemic regions of Europe and Asia (1). LD spreading depends on geographic, environmental and climatic factors, and on the pathogenicity of Borrelia burgdorferi sensu lato (s.l.) (2).

LD is a multisystem disorder caused by several genotypic species B. burgdorferi s.l. complex (3). The complex includes at least 20 species, but the main pathogenic genomic species responsible for human LD in Central Europe and Eastern Europe are $B$. burgdorferi sensu stricto, $B$. garinii and B. afzelii. However, the DNA from $B$. valaisiana, $B$. lusitaniae and $B$. spielmani detected in samples of human origin or spirochetes was isolated from patients with LD symptoms (4).

The only clinical manifestation sufficient to diagnose LD without laboratory confirmation is the identification of erythema migrans (EM) rash after ticking. The CDC defined the EM rash as an expand- ing red macula or papule, which must be at least $5 \mathrm{~cm}$ (with or without central cleaning) (5).

Available laboratory methods for LD diagnosis can be divided into two categories - direct methods (cultivation or techniques that detect specific proteins or nucleic acids specific for $B$. burgdorferi) and indirect methods (serology for antibody detection) (6).

Laboratory tests for direct detection of $B$. burgdorferi are limited by the very low number of spirochetes in most clinical specimens. The most common evidence of infection is the direct cultivation of Borrelia burgdorferi s.l. or the PCR method (7). Cultivation of the pathogen is successful in 50-70\% of cases in skin lesion patients, but only in 10-30\% of CSF in patients with neuroborreliosis. PCR results are similar. Concentration of Borrelia in peripheral blood is relatively low, more suitable is EM skin biopsy (50-70\% positivity), synovial fluid from the affected joint $(50-70 \%$ positivity), CSF ( $10-30 \%$ positivity) (7).

Serological tests are often misinterpreted and may be false positive in results unless validated laboratory tests are performed (8). The Center for Disease Control and Prevention (CDC) and European Concerted 
Action on Lyme Borreliosis (EUCALB) recommends a two-stage serological protocol using an enzyme-linked immunosorbent assay followed by a more speculative Western blot/immunoblot to confirm the diagnosis when the test samples are positive or ambiguous (5).

The sensitivity of the antibody-based assays increases with duration of the infection and there will be a delay from the initial infection until sufficient antibody levels are found. Patients who have a very early illness have a rather negative result. Serological tests are useful in patients with clinical findings suggesting later stages of LD (7).

The aim of the presented research was to analyze the incidence of LD based on the presence of specific antibodies in the serum of patients in Eastern Slovakia and to compare the results of serological ELISA and immunoblot assays.

\section{MATERIALS AND METHODS}

\section{Study Design}

Study design: cross-sectional study. Approval of the study protocol was received by the Ethics Committee of the University Hospital of Košice.

\section{Data Collection}

The study was conducted from 2013 to 2016. Blood samples were obtained from 537 participants ( 287 men and 250 women) from nonrandom sampling. The study included people in whom we predicted possible symptoms associated with Lyme borreliosis - people with work activities in nature, patients from different clinical departments and the population of marginalized people (Roma ethnicity).

\section{Serum Samples}

The serum was obtained by the centrifugation ( $2500 \mathrm{rpm} / 10 \mathrm{~min}$ ) and preserved at $-80{ }^{\circ} \mathrm{C}$ until serological testing. The sera were processed in the Laboratory of the Department of Microbiology and Immunology of the University of Veterinary Medicine and Pharmacy in Košice.

\section{Questionnaire}

The subjects were personally interviewed according to a structured questionnaire specifically designed for the study. Three main categories of variables were considered in the questionnaire: demographic data (age, gender and residence), epidemiological data (tick bites, insect bites, pet ownership, frequent outdoor activities) and clinical data (in the past occurring diseases/symptoms).

\section{Laboratory Analysis}

Using the ELISA method, we examined 537 human sera and, for confirmation, we found all positive IgG antibodies against Borrelia by immunoblot assay. The assay was performed with commercial ELISA assays for $\mathrm{IgG} / \mathrm{IgM}$ (recombinant) (NovaLisa ${ }^{\mathrm{TM}}$, Germany) according to the manufacturer's instructions. Results equal to $11 \mathrm{NTU} / \mathrm{mL}$ and above were considered positive, values between 9 and $10 \mathrm{NTU} / \mathrm{mL}$ were considered borderline and values below $9 \mathrm{NTU} / \mathrm{mL}$ were considered negative. The presence of $\mathrm{IgG}$ antibodies against the specific antigen Borrelia burgdorferi s.l. was determined by immunoblot assay using commercial LYMECHECK OPTIMA IgG and IgM kits (BIOSYNEX, France). In kits, recombinant proteins are specific for 5 genus species: B. burgdorferi s.s., B. afzelii, B. garinii, B. spielmanii and B. bavariensis. The tests were carried out in accordance with the procedure recommended by the manufacturer. Positive or negative results were determined based on the sum of the point values assigned to the individual bands corresponding to the reaction of $\mathrm{IgG}$ antibodies with specific B. burgdorferi antigens. Results of $\leq 5$ were considered negative, those of 6 were classified as borderline and those of $>7$ were considered positive.

\section{Statistical Analysis}

Categorical variables were given as percentage $(\mathrm{n} / \mathrm{N})$ and compared by Pearson chi-square test. Logistic regression was employed to evaluate the association of seroprevalence with the influence factors of LD. Value considered for statistical significance was $\mathrm{p}<0.05$. All data were analyzed with the SPSS software package (Version 21.0).

\section{RESULTS}

\section{File Characteristics}

The study group consisted of 537 people aged between 12 to 93 (average age 57.05 years, SD 16.29), which included 287 (53.4\%) men and $250(46.6 \%)$ women. The average age of men was 45.2 (SD 16.04 years) and the average age of women was 54.8 (SD 16.94 years). Urban residence was reported by $281(52.3 \%)$ participants and rural by 256 (47.7\%) (Table 1).

According to the questionnaire results, of these 537 people, $154(28.7 \%)$ rheumatological, 127 (23.6\%) neurological and $78(14.5 \%)$ dermatological complaints were observed. The presence of selected symptoms over the last 5 years was statistically more pronounced in women compared to men $(\mathrm{p}<0.05)($ Table 2$)$.

\section{General Screening}

The B. burgdorferi screening ELISA test revealed 425 (79.1\%) negative samples for anti-B. burgdorferi s.l. IgG, whereas $16(3.0 \%)$ samples were borderline and $96(17.9 \%)$ were positive. The results for IgM were as follows: 479 negative (89.2\%), 17 (3.2\%) borderline and $41(7.6 \%)$ positive samples. The immunoblot, which was performed for the positive IgG samples, was positive in 67 subjects $(69.8 \%)$, borderline results were detected in $8(8.3 \%)$ subjects and negative in $21(21.9 \%)$ subjects of the IgG samples. More positive samples were found out by ELISA method compared to immunoblot. The detailed results about the classes of the detected antibodies are presented in Figure 1.

Most commonly we detected antibodies against VIsE (80.2\%), p41 (66.7\%), p18 (56.3\%), p100 (41.7\%), p58 (31.3\%), and p39 $(30.2 \%)$ by immunoblot assay. The B. afzelii $(54.2 \%)$ and $B$. bavariensis (13.5\%) were the most frequent cause of the immunological reaction of all analyzed antigens. The B. spielmanii and $B$. burgdorferi sensu stricto antigens were rarely detected (1.0\% and 4.2\%) (Fig. 2). 
Table 1. General characteristics of examined subjects $(N=537)$

\begin{tabular}{|l|l|c|c|c|c|c|c|}
\hline \multicolumn{2}{|c|}{} & \multicolumn{2}{c|}{ Male } & \multicolumn{2}{c|}{ Female } & \multicolumn{2}{c|}{ Total } \\
\cline { 3 - 8 } \multicolumn{2}{|c|}{} & $\mathbf{n}$ & $\%$ & $\mathbf{n}$ & $\%$ & $\mathbf{n}$ & $\%$ \\
\hline \multicolumn{2}{|l|}{ Age-Mean (SD) } & - & $45.2(16.04)$ & - & $54.8(16.94)$ & - & $57.05(16.29)$ \\
\hline \multirow{2}{*}{ Residence } & urban & 144 & 50.2 & 137 & 54.8 & 281 & 52.3 \\
\cline { 2 - 8 } & rural & 143 & 49.8 & 113 & 45.2 & 256 & 47.7 \\
\hline \multicolumn{2}{|l|}{ Total number } & 287 & 53.4 & 250 & 46.6 & 537 & 100 \\
\hline
\end{tabular}

Table 2. Comparison of intersexual differences in frequency of presence of selected symptoms of diseases $(N=537)$

\begin{tabular}{|c|c|c|c|c|c|}
\hline Parameter & & $\begin{array}{l}\text { Men } \\
\mathrm{n}(\%)\end{array}$ & $\begin{array}{c}\text { Women } \\
\mathrm{n}(\%)\end{array}$ & $\begin{array}{l}\text { Total } \\
\mathrm{n}(\%)\end{array}$ & $\mathrm{p}$-value \\
\hline \multicolumn{6}{|c|}{ The presence of symptoms in the last 5 years } \\
\hline \multirow{2}{*}{ Neurological } & yes & $55(19.2)$ & $72(28.8)$ & $127(23.6)$ & \multirow{2}{*}{$<0.01$} \\
\hline & no & $232(80.8)$ & $178(71.2)$ & $410(76.4)$ & \\
\hline \multirow{2}{*}{ Rheumatological } & yes & $54(18.8)$ & $100(40.0)$ & $154(28.7)$ & \multirow{2}{*}{$<0.001$} \\
\hline & no & $233(81.2)$ & $150(60.0)$ & $383(71.3)$ & \\
\hline \multirow{2}{*}{ Dermatological } & yes & $31(10.8)$ & $47(18.8)$ & $78(14.5)$ & \multirow{2}{*}{$<0.01$} \\
\hline & no & $256(89.2)$ & $203(81.2)$ & $459(85.5)$ & \\
\hline
\end{tabular}

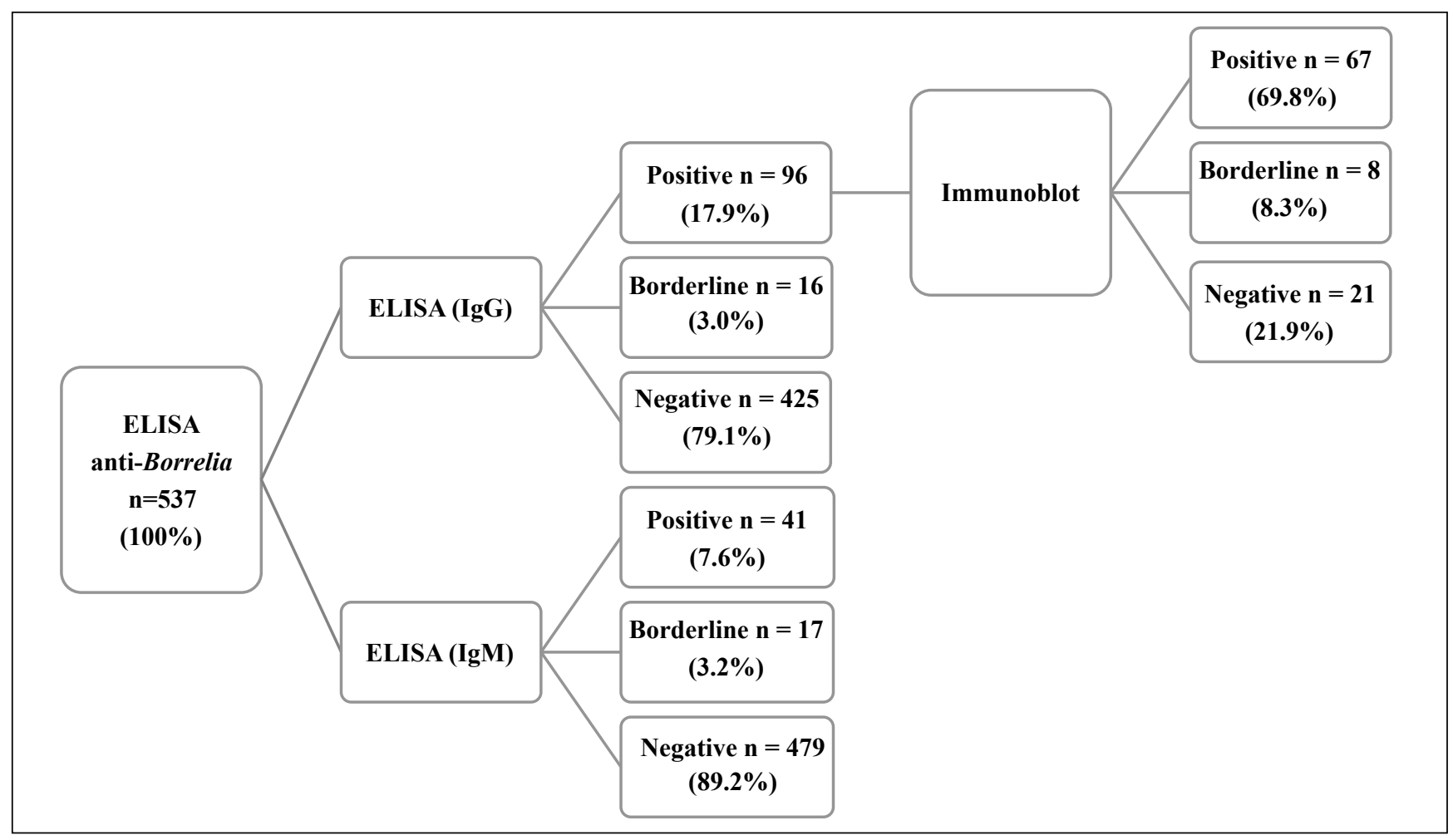

Fig. 1. Results of serology of IgG/lgM antibodies against B. burgdorferi s.l. by ELISA and immunoblot.

We did not confirm a statistically significant difference $(p>0.05)$ between males and females in the range of antibodies against individual antigens in our set, but men showed a higher percentage of antibodies than women almost for each antigen (Fig. 3).

We have not confirmed a significant relationship between the presence of antibodies against the p39, B. afzelii and B. burgdorferi s.s. within relation to selected symptoms (neurological, rheumatological and dermatological) $(\mathrm{p}>0.05)$ (Table 3).

\section{DISCUSSION}

Slovakia, like other European countries, is exposed to an increased risk of LD, which can also occur in non-endemic areas. Human LD is becoming more and more frequent. According to the Public Health Office of the Slovak Republic in 2017, 806 LD cases were reported in Slovakia. The highest occurrence was observed in the Banská Bystrica, Žilina and Trenčín regions (9). 


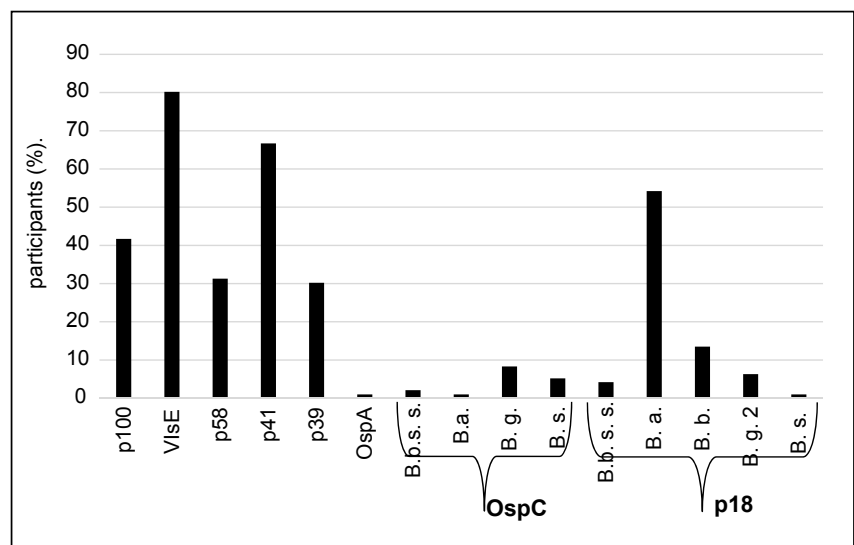

Fig. 2. Percentage of respondents with IgG anti-specific antigens B. burgdorferi s.l.

p100 - cytoplasmic protein; VIsE - variable major protein like sequence; p58 - protein of the membrane; p41 - flagellin protein; p39 - specific protein of the membrane; OspA/C - outer surface protein A/C; p18 - surface protein;

B.a. - B. afzelii; B.g. - B. garinii; B.b.s.s. - B. burgdorferi s.s.; B.s. - B. spielmanii

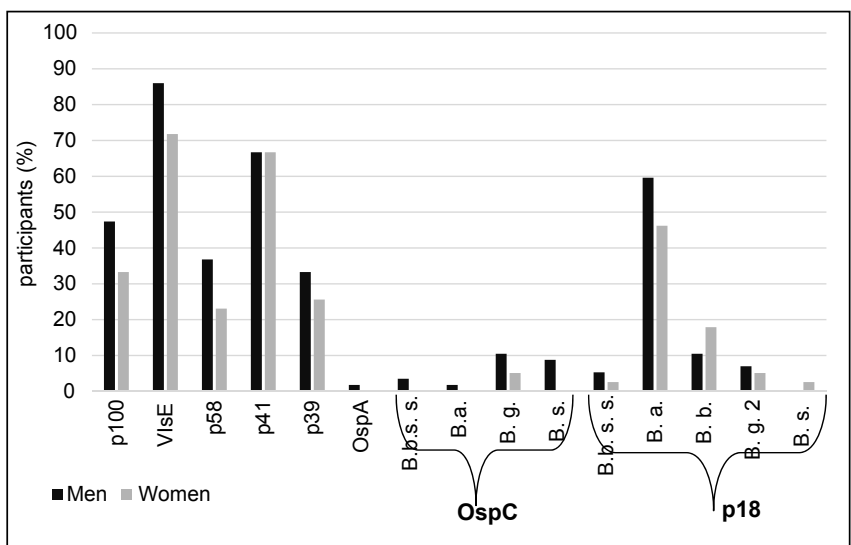

Fig. 3. Comparison of intersexual differences in IgG antibody response against individual antigens $B$. burgdorferi s.l.

p100 - cytoplasmic protein; VIsE - variable major protein like sequence;

p58 - protein of the membrane; p41 - flagellin protein; p39 - specific protein of the membrane; OspA/C - outer surface protein A/C; p18 - surface protein;

B.a. - B. afzelii; B.g. - B. garinii; B.b.s.s. - B burgdorferi s.s.; B.s. - B. spielmanii

Table 3. Logistic regression of the effect of antibodies against B. afzelii, B. burgdorferi s.s. and p39 antigen in relation to selected symptoms

\begin{tabular}{|l|c|c|c|c|c|c|}
\hline \multirow{2}{*}{ Factor } & \multicolumn{2}{|c|}{ Neurological } & \multicolumn{2}{c|}{ Rheumatological } & \multicolumn{2}{c|}{ Dermatological } \\
\cline { 2 - 7 } & OR $(95 \% \mathrm{Cl})$ & p-value & OR $(95 \% \mathrm{Cl})$ & p-value & OR $(95 \%$ Cl) & p-value \\
\hline B. afzelii & $2.09(0.76-5.71)$ & n.s. & $1.39(0.54-3.62)$ & n.s. & $1.39(0.54-4.03)$ & n.s. \\
\hline B. burgdorferi s.s. & $1.11(0.11-11.25)$ & n.s. & & - & & - \\
\hline p39 & $0.58(0.19-1.76)$ & n.s. & $0.62(0.21-1.89)$ & n.s. & $0.44(0.12-1.66)$ & n.s. \\
\hline
\end{tabular}

OR - Odds ratio; $\mathrm{Cl}$ - confidence interval; n.s. - not significant $(\mathrm{p}>0.05)$

The most frequently used laboratory method for LD diagnosis is serology using the two-tier (Enzyme Immunoassay - ELISA followed by Western blot - WB) algorithm. This algorithm, when using specific interpretation criteria, is the method recommended by infectious disease experts and public health organizations (10).

Antibodies determination from serum and CSF by ELISA is one of indirect methods with high sensitivity but low specificity. This is due to the high seroprevalence (10-20\% of the adult population), the incidence of cross-reactive antibodies and the occurrence of seronegative forms, which in some cases is associated with the risk of misdiagnosis (11).

The limitation of our study is that we did not perform immunoblot assays by screening serum for IgG bound antibodies and anti-IgM antibodies. Another limitation was a small group of respondents (predominantly people from Eastern Slovakia).

Our results confirmed a high sero-prevalence of anti-Borrelia antibodies (17.9\% for IgG), while the immunoblot seropositive test was confirmed in $69.8 \%$ of respondents from ELISA positive sera against IgG. Positive IgM class antibodies were found in $7.6 \%$ of studied population. Higher seroprevalence may be associated with the increasing risk of infestation with infected ticks in Eastern Slovakia as well. Similar results were obtained by the first studies conducted in Poland, which found the presence of ELISA antibodies at $11-13 \%(12)$.

Since the immunoblot method offers the possibility of examining the reactivity of the individual antigenic fractions, the positive results were further analyzed and compared.
The diagnostic accuracy of ELISA and immunoblot for LD in Europe varies considerably, with an average sensitivity of $\sim 80 \%$ and a specificity of $\sim 95 \%$. Nineteen case control studies, including healthy controls, evaluated the accuracy of serological tests at EM. The sensitivity for ELISA or immunoblot at EM was 50\% and specificity $95 \%$. ELISA tests were more accurate than immunoblots, mainly due to higher sensitivity. For Lyme arthritis, the sensitivity was approximately $95 \%$ and in the cross-sectional studies of neuroborreliosis the specificity decreased to $78 \%$ (13). In studies conducted in Turkey in 2005 for groups of farmers and forest workers, the ELISA test for IgG class antibodies showed seroprevalence between $10.9 \%$, while Western blot confirmed positive only in $1.1 \%$ (14).

In patients with early phase $\mathrm{LD}$, the most commonly detected antibodies are IgM against flagelin protein (p41) and outer surface protein $\mathrm{C}(\mathrm{OspC})$. Many studies have also been confirmed in Poland $(4,15,16)$.

In our study, we detected IgG flagella protein p41 in $66.7 \%$ of individuals. Other antibodies with respect to high frequency were antibodies against VIsE (80.2\%), p100 (41.7\%), p39 (30.2\%), p58 (31.3\%) and p18 for B. afzelii (54.2\%) that are associated with serum IgG antibodies with a later stage of infection. It is a higher percentage than in the study by Kubiak et al., where antibodies against VlsE protein were detected in $64.8 \%$, antibodies against p18 and p100 B. afzelii in $24.1 \%$ (17). OspA antibodies (1\%) also rarely occurred in our study. In the study by Jovanovic et al., IgG showed a high reactivity against VIsE, p19, p41, OspC, OspA and p17 (3). In the study by Tokarska-Rodak et al., VIsE was detected in only $40 \%$ and p39 in $29 \%$ of the samples (4). VlsE is considered 
the most sensitive recombinant $B$. burgdorferi antigen and used in diagnostics. Antibodies to this protein can be found in serum even 6 months after successful treatment with antibiotics (3).

In these tests, besides the antigens specific for $B$. burgdorferi S.s, B. afzelii and B. garinii-species that dominate the infections in Europe - there are also species that less often cause LD (such as $B$. spielmanii) (17). According to genospecies, borrelia is characterized by a certain organ tropism. It has been proved that $B$. burgdorferi s.s. most often cause arthritic symptoms, while $B$. afzelii is the most common in patients with skin symptoms of LD - erythema migrans (EM) and atrophic acrodermatitis $(17,18)$. By logistic regression, we have not been able to detect a significant relationship between the presence of B. afzelia and B. burgdorferi s.s. and selected diseases $(p>0.05)$. The presence of the $\mathrm{p} 39$ antigen is often associated with arthritic symptoms (3). In our work p39 was not a significant factor for the presence of selected diseases. In the study, we detected a strong reaction with $B$. spielman at $1 \%$, which is lower than in the study by Kubiak et al. (17) and Biesiada et al. (15), where they identified the response in $4.3 \%$ and $8 \%$ of individuals, respectively.

Type of antibodies of each class in serum depended on heterogeneity species $B$. burgdorferi s.l. a complex cause infections and the polymorphism of their antigens. Recombinant proteins used in immunoblot as diagnostic antigens are not only highly immunogenic properties but are also selected to indicate stage of disease progression (19).

The diagnosis of LD, especially in the absence of the characteristic rash, may be difficult, since the other clinical manifestations of LD are not specific. Even the diagnosis of EM sometimes may be difficult, since the rash initially may be confused with nummular eczema, granuloma annulare, an insect bite, ringworm, or cellulitis (6).

The current 2-tier algorithm works relatively well when used as recommended, but problems include the low sensitivity during early infection, subjective interpretation of bands, and confusion by healthcare providers and patients regarding how to interpret results.

\section{CONCLUSION}

In summary, it should be noted that the detection of antibodies against B. burgdorferi s.l. is only an indirect evidence of the participation of this bacterium in causing clinical symptoms of LD in human. The standard diagnostic procedure of LD is quite simple if a positive history of tick exposure or typical EM appears. Lack of unequivocal clinical symptoms creates the necessity for further evaluation with laboratory tests such as ELISA and immunoblot. Final confirmation would require detection and genome identification B. burgdorferi s.l. in the material obtained directly from the patient and in the local population of $I$. ricinus using methods of molecular biology (17).

\section{Acknowledgements}

This work was supported by Grants VEGA No. 1/0198/13, 1/0011/14 of the Ministry of Education, Science, Research and Sport of the Slovak Republic and project VVGS -3/GSD/2016. We also thank the Board of Directors of University Hospital in Košice for assistance with organization of sample collection at the hospital wards.

\section{Conflict of Interests}

None declared

\section{REFERENCES}

1. Tomao P, Ciceroni L, D’Ovidio MC, De Rosa M, Vonesch N, Iavicoli S, et al. Prevalence and incidence of antibodies to Borrelia burgdorferi and to tick-borne encephalitis virus in agricultural and forestry workers from Tuscany, Italy. Eur J Clin Microbiol Infect Dis. 2005;24(7):457-63.

2. Medlock JM, Hansford KM, Bormane A, Derdakova M, Estrada-Peña A, George J-C, et al. Driving forces for changes in geographical distribution of Ixodes ricinus ticks in Europe. Parasit Vectors. 2013;6:1.

3. Jovanovic D, Atanasievska S, Protic-Djokic V, Rakic U, Lukac-Radoncic E, Ristanovic E. Seroprevalence of Borrelia burgdorferi in occupationally exposed persons in the Belgrade area, Serbia. Braz J Microbiol. 2015;46(3):807-14.

4. Tokarska-Rodak M, Fota-Markowska H, Kozioł-Montewka M, Śmiechowicz F, Modrzewska R. The detection of specific antibodies against B. burgdorferi s. s., B. afzelii, B. garinii, and B. spielmani antigens in patients with Lyme disease in eastern Poland. New Med. 2010;3:84-7.

5. Wright WF, Riedel DJ, Talwani R, Gilliam BL. Diagnosis and management of Lyme disease. Am Fam Physician. 2012;85(11):1086-93.

6. Dorko E. Lyme borreliosis, characteristics, epidemiology, treatment, prevention, environmental and social aspects of occurrence. In: Rimárová K, Barlašová-Dunajová M, Bušová A, Diabelková J, Dorko E, Hamráková M, et al. Selected health parametres of the Romany Gypsy population in the Slovak Republic. Košice: Equlibria; 2017. p. 230-62. (In Slovak.)

7. Marques, AR. Laboratory diagnosis of Lyme disease - advances and challenges. Infect Dis Clin North Am. 2015;29(2):295-307.

8. Borchers AT, Keenb CL, Huntleyc AC, Gershwin ME. Lyme disease a rigorous review of diagnostic criteria and treatment. J Autoimmun. 2015; 57:82-115.

9. Public Health Authorities of the Slovak Republic. Annual report [Internet]. Bratislava: Public Health Authorities of the Slovak Republic; 2017 [cited 2018 May 27]. Available from: http://www.uvzsr.sk/docs/ vs/vyrocna sprava SR 2017.pdf/. (In Slovak).

10. Ogden NH, Arsenault J, Hatchette TF, Mechai S, Lindsay LR. Antibody responses to Borrelia burgdorferi detected by western blot vary geographically in Canada. PLoS One. 2017;12(2):e0171731.

11. Bil-Lula I, Matuszek P, Pfeiffer T, Woźniak M. Lyme Borreliosis - the Utility of Improved Real-Time PCR Assay in the Detection of Borrelia burgdorferi Infections. Adv Clin Exp Med. 2015;24(4):663-70.

12. Pańczuk A, Koziol-Montewka M, Tokarska-Rodak M. Exposure to ticks and seroprevalence of Borrelia burgdorferi among a healthy young population living in the area of southern Podlasie, Poland. Ann Agric Environ Med. 2014;21(3):512-17.

13. Leeflang MM, Ang CW, Berkhout J, Bijlmer HA, Van Bortel W, Brandenburg AH, et al. The diagnostic accuracy of serological tests for Lyme borreliosis in Europe: a systematic review and meta-analysis. BMC Infect Dis. 2016;16:140.

14. Kaya DA, Parlak HA, Ozturk EC, Behcet M. Seroprevalence of Borrelia burgdorferi infection among forestry workers and farmers in Duzce, northwestern Turkey. New Microbiol. 2008;(31):203-9.

15. Biesiada G, Czepiel J, Salamon D, Garlicki A, Dziubek A, Maziarz B, et al. Analysis of Borrelia burgdorferi genostrains among patients with Lyme disease. Przegl Lek. 2009;66:511-2. (In Polish).

16. Wojciechowska-Koszko I, Mączyńska I, Szych Z, Giedrys-Kalemba S Serodiagnosis of borreliosis: indirect immunofluorescence assay, enzymelinked immunosorbent assay and immunoblotting. Arch Immunol Ther Exp (Warsz). 2011;59(1):69-77.

17. Kubiak K, Dzika E, Równiak J, Dziedziech M, Dzisko J. Seroprevalence of Lyme disease and genospecies of Borrelia burgdorferi sensu lato in patients diagnosed with borreliosis in the Province of Warmia-Masuria in north-eastern Poland. Ann Agric Environ Med. 2012;19(2):203-7.

18. van Dam AP, Kuiper H, Vos K, Widjojokusumo A, de Jongh BM, Spanjaard L, et al. Different genospecies of Borrelia burgdorferi are associated with distinct clinical manifestations of Lyme borreliosis. Clin Infect Dis. 1993;17(4):708-17.

19. Sicklinger $M$, Wienecke R, Neubert U. In vitro susceptibility testing for four antibiotics against Borrelia burgdorferi: a comparison of results for the three genospecies Borrelia afzelii, Borrelia garinii, and Borrelia burgdorferi sensu stricto. J Clin Microbiol. 2003;41(4):1791-3. 